\title{
In-line NDT with X-Ray CT combining sample rotation and translation
}

Thomas De Schryveria, Jelle Dhaene ${ }^{\mathrm{a}}$, Manuel Dierick ${ }^{\mathrm{a}}$, Matthieu N. Boone $^{\mathrm{a}}$, Eline Janssens ${ }^{\mathrm{b}}$, Jan Sijbers ${ }^{\mathrm{b}}$, Mattias van Dael $^{\mathrm{c}}$, Pieter Verboven ${ }^{\mathrm{c}}$, Bart Nicolai ${ }^{c}$, Luc Van Hoorebeke ${ }^{a}$

${ }^{a} U G C T$, Dept. Physics and Astronomy, Ghent University, Proeftuinstraat 86, Ghent, 9000, Belgium

${ }^{b}$ iMinds-VisionLab, Dept. of Physics, University of Antwerp, Universiteitsplein 1, Antwerp, 2610, Belgium

${ }^{c}$ MeBios, Dept. of Biosystems, KU Leuven, W. de Croylaan 42, Heverlee, 3001, Belgium

\begin{abstract}
An alternative acquisition geometry for X-ray computed tomography (CT) is investigated as a solution to in-line non-destructive quality inspection in a high throughput production environment. The sample movement during acquisition combines a translation, typically horizontal, along one axis and a rotation about a second axis perpendicular to the first, and is shown to produce theoretically exact CT reconstructions. A methodology is presented to evaluate the design of a conveyor belt implementation for this acquisition scheme, investigating the trade-off between reconstruction quality and throughput. The methodology was applied in both a simulated version and an experimental mock-up of the conveyor belt implementation for a specific food sample, but can be extrapolated to any type of sample. Throughput, for the food sample, is predicted to be in a practically usable range of up to 5 samples per second. As a general conclusion, higher throughput can be reached with larger inspection stations while maintaining image quality.
\end{abstract}

Keywords: X-ray tomography, in-line, inspection systems, iterative reconstruction

\footnotetext{
${ }^{1}$ Corresponding author: E-mail thomas.deschryver@ugent.be Tel. +329264 6628 Fax +3292646697
} 


\section{Introduction}

X-ray transmission has become a valuable tool in many industrial branches to ensure the quality of a product through non-destructive evaluation. A first way to image a product's interior is through simple 2D radiographic etion, in which all features on the inside and outside of a between an X-ray source and a detector are superimposed on one single image. Although some internal defects cannot be distinguished on these single point of view projection images, it is a fast way of imaging the interior of a product in-line and is used as such in a wide variety of application inspection in electronics and contaminant detection in food products 1 5]. In X-ray computed tomography (CT) a series of these 2D radiographs, taken from multiple directions, can be combined to reconstruct a full 3D visualization of an object's interior. While CT has proven to be successful itself should not compromise the desired production throughput and at the same time preserve enough image quality to ensure defect detectability. In other words, the image acquisition should be as fast as possible, which is attained by lowering the X-ray detector's exposure time and by acquiring less

projections is acquired to accurately reconstruct all of the interesting features in the interior of the scanned object. For the food industry in particular, some defects, such as browning disorders in fruit, inherently show low contrast with respect to their surroundings and are often very small, consequently requiring between a high acquisition speed and a high contrast and resolution image, is one of the main reasons why 3D X-ray CT has not yet touched ground as an inspection tool in food industry. CT is already used in-line or rather atline in other industrial branches, primarily as a metrology tool for inspecting as an explosive detection system by airport security. Most of these systems can either be categorized as batch delivery or pick-and-place systems [7, 8], where the samples are scanned one by one, or as continuous throughput conveyor belt systems using a helical scanning approach, similar to medical

35 CT equipment [9]. In a notable new development the rotating gantry used in these helical systems is being replaced by a series of stationary sources and detectors placed on a circle around the conveyor belt [10, 11]. 
In this work we consider a conveyor belt setup where an object passes between a stationary X-ray source on one side and a fixed large flat panel detector on the other side, while also performing a rotational movement. Theoretically a complete angular sampling is only possible for the points in the central plane of the object, whereas off-centre points cannot be reconstructed exactly, progressively showing more and more cone beam artefacts as the distance from the central slice increases. However, the projections fications and a small cone beam angle, resulting in a low expression of its accompanying cone beam artefacts in the vertical direction. The simplicity of the conveyor belt geometry does make it a potential candidate for in-line inspection and a possible competitor for helical scanning geometries, which do offer a complete angular sampling of the entire object but are often more complex in their implementation.

While the focus here is on CT acquisition, the imaging chain does not stop there. First of all, a valid 3D inspection and defect detection requires the 2D radiographic projections to be reconstructed into a 3D volume. Secondly, the $3 \mathrm{D}$ volumes have to be analysed to extract the relevant information about possible defects. The reconstruction and imaging analysis encompass a computational effort, which has to be addressed with the right computing power and clever algorithms. Improvements in these downstream imaging steps can however be very application dependent, which does not fit into the general study on the performance of the conveyor belt geometry. On the other hand, these improvements might greatly relax the demands on acquisition, e.g. by significantly lowering the amount of projections and the X-ray dose, and thus the SNR, needed for a good reconstruction of an object's interior [12 14]. In this work the acquisition is handled separately, with a particular emphasis on antee qualitative reconstruction with a standard reconstruction technique.

It is the aim of this work to establish the feasibility of performing inline CT inspections with and to define geometric design constraints for the conveyor belt geometry. In section 2 this conveyor belt geometry is discussed configuration of the setup, using a realistic Elstar apple phantom in sections 3.1 and 3.2 . The quality of the 3D reconstructions is quantified through the Spectral Signal to Noise Ratio $(S S N R)$, as introduced in section 3.3, and set out against the expected throughput for the configuration at hand. In conclusion, some general guidelines for the dimensions of the imaging setup 
and the number of required projections are formulated, based on plots of quality in terms of throughput.

\section{Theory}

\subsection{Geometrical constraints for an exact conveyor belt acquisition}

In figure 1 a schematic top view of the conveyor belt setup is shown. An X-ray source point is kept at a fixed distance (Source Detector Distance, $S D D$ ) from a large flat panel detector. The sample itself performs a translation from the left to the right at a fixed distance from the source (Source Object Distance, $S O D$ ), parallel to the central row of the detector and in the plane containing both this central detector row and the source point. While traveling a horizontal distance $(H)$, the sample also performs a rotational movement as a supplement to the inherent change in parallax related to the translational movement. This is most efficiently realized with a counter clockwise rotation as viewed from the top.

The angle for this rotation has to be chosen such that every point in the sample is intersected by a source ray over an angular range of at least $180^{\circ}$. In computed tomography this condition is more commonly referred to as the Tuy-Smith condition [15] leading to a so-called complete trajectory. Formulated in a more intuitive way, the Tuy-Smith condition requires that relative to any given point in the object the source traces out a trajectory in space which does not intersect the given point and for which the trajectory's end points are collinear with this given point. The resulting trajectory will henceforth be referred to as complete and for a conventional circular cone beam scan this amounts to a rotation of

$$
180^{\circ}+2 \gamma
$$

where $2 \gamma$ is the so-called fan angle of a wedge originating from the source point, and which completely covers the scanned object. For a conveyor belt geometry, the rotation angle forming a complete trajectory can be found by considering the object at its central position between the source and the detector, i.e. halfway its translation where the central ray cuts the region of interest (ROI) into two symmetric halves (see figure 1). For now, the ROI is considered to be circular, but as shown in figure 4 its shape can be extended. Any conclusion regarding the covered angular range for a point in the right half of this ROI w.r.t. its movement on the left side of the source is equivalent 


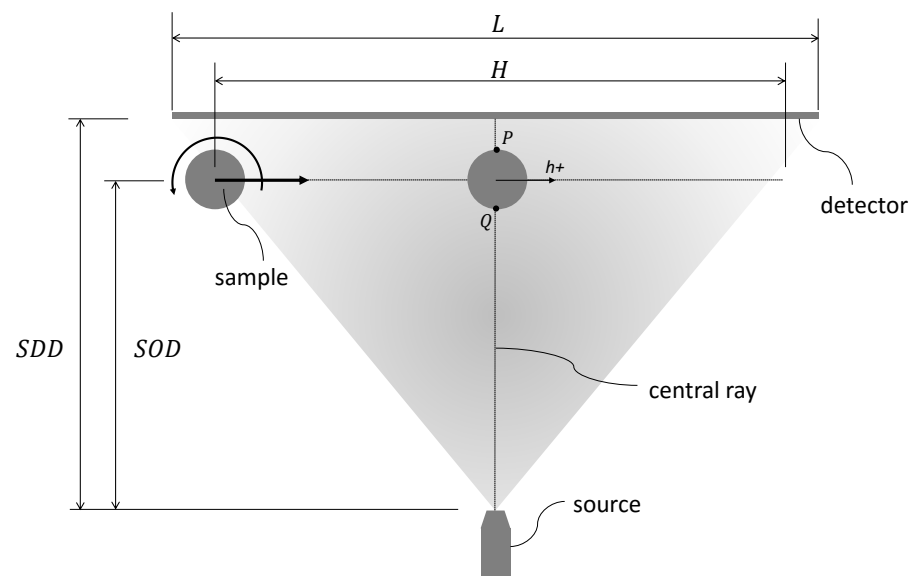

Figure 1: Schematic top view of the conveyor belt geometry. The sample combines a rotation and a translation over a distance $H$ at a fixed distance from the source, $S O D$. The detector is kept at a distance $S D D$ from the source.

to the symmetric case for a point in the left half w.r.t. its movement on the right side of the source. Furthermore, it can be shown (see figure 4) that the points on the intersection between the outer rim on the ROI and the central ray, indicated by $P$ and $Q$ on figures 1 and 2 , will cover the smallest angular range of all points within the ROI. The fact that these points have to be sampled from at least $180^{\circ}$ puts forward two conditions connecting the a priori chosen geometrical parameters, in most practical cases the detector length $(L)$, the Object Detector Distance $(O D D)$ and the ROI diameter $\left(D_{R O I}\right)$ and the source's half opening angle $(\alpha)$, to the a priori unknown parameters, i.e. the $S O D, S D D$, the detector run-out $(W)$ and the half rotation angle $(\Theta)$ as defined by figure 2 .

1. While moving backward from its central position, the point $Q$ will be the first point to be projected out of the detector field. At this outer position the source rays should have covered at least $90^{\circ}$ around point $Q$. This condition occurs when the ROI is tangent to the outer ray in $Q$ as depicted in figure 2(a) and connects $\alpha$ to $W, D_{s}$ and the $O D D$ :

$$
\frac{D_{R O I}}{\cos (\alpha)}=W-2 O D D \tan (\alpha)
$$

The opening angle itself is given by

$$
\frac{L}{2 S D D}=\tan (\alpha)
$$




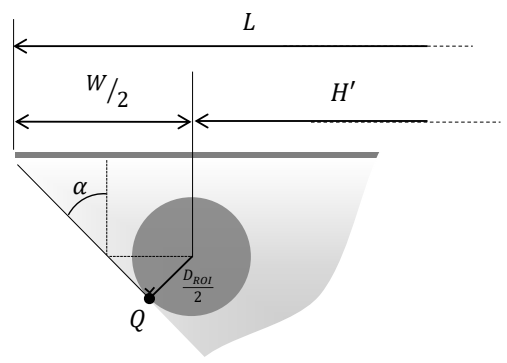

(a)

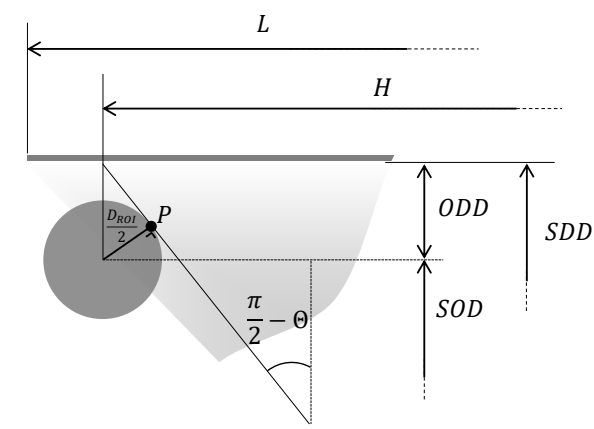

(b)

Figure 2: (a) Condition 1: In point $Q$, the ROI is tangent to the outer ray, which connects the source point to the detector edge. (b) Condition 2: The ROI has a tangent source ray in point $P$.

2. To complete the trajectory for point $P$ there has to be a source ray tangent to the ROI in $P$ (see figure 2(b)). With $\pi / 2-\Theta$ being the angle between this ray and the central source ray, the following condition holds:

$$
H=\frac{1}{\sin (\Theta)}\left(2 S O D \cos (\Theta)+D_{R O I}\right)
$$

Keeping in mind that

$$
S D D=S O D+O D D
$$

and using equation 2, equation 1 can be reformulated as

$$
H^{\prime}=L-W=\frac{1}{\sin \left(\Theta^{\prime}\right)}\left(2 S O D \cos \left(\Theta^{\prime}\right)-D_{R O I}\right)
$$

with $\alpha=\pi / 2-\Theta^{\prime}$. Finally, equations 1 to 5 can be combined to form the following set

$$
\begin{aligned}
S D D & =\frac{L}{2 \tan (\alpha)} \\
S O D & =S D D-O D D \\
\frac{\Theta^{\prime}}{\Theta} & =\frac{\sin (\Theta)}{\sin \left(\Theta^{\prime}\right)} \cdot \frac{2 S O D \cos \left(\Theta^{\prime}\right)-D_{R O I}}{2 S O D \cos (\Theta)+D_{R O I}}
\end{aligned}
$$


where we have implicitly assumed that

$$
\frac{\Theta^{\prime}}{\Theta}=\frac{H^{\prime}}{H}
$$

110 lation and as such evolves linearly with it. In general, when considering a counter clockwise rotation, the rotation angle can follow any continuous mapping along the horizontal travel abiding to certain restrictions as discussed in paragraph 5.2 .

115 Using equations 6 to 8 , the $S D D, S O D$ and $\Theta$ can be calculated, given $L, \alpha, O D D$ and $D_{R O I}$, which fixes the entire conveyor belt geometry. In figure 3, the trajectories for the points $P$ and $Q$ are plotted for a situation in which all of the geometrical parameters comply to equations 6 to 8 , with $L=966 \mathrm{~mm}, \alpha=40^{\circ}, O D D=75 \mathrm{~mm}$ and $D_{R O I}=90 \mathrm{~mm}$ (corresponding$$
\text { containing the ROI where calculated for this specific situation in figure } 4(\text { a) }
$$
containing the ROI where calculated for this specific situation in figure 4(a), The contour containing the points which cover at least $180^{\circ}$, is indicated by a thick black line. Theoretically, all the points within this contour can be reconstructed exactly, in particular the points from the circular ROI conthe dashed line in figure 4(a), it can be seen that the points $P$ and $Q$ do indeed cover an angular range of $180^{\circ}$, while the other points on the ROI circumference cover a larger range.

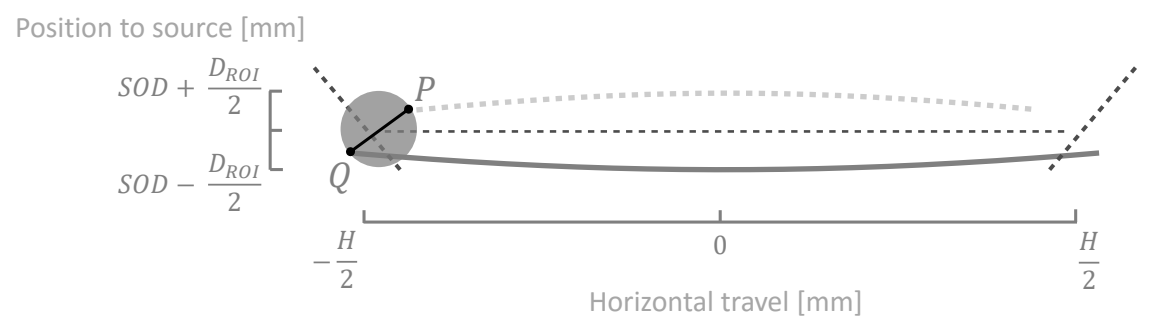

Figure 3: Trajectories of the points $P$ and $Q$ throughout their conveyor belt movement. The ROI is drawn at the start of the acquisition, i.e. at the moment condition 2 for a complete trajectory holds. It should be noted that the object is only mildly rotated, i.e. $111^{\circ}$ for the specific case depicted here, with $L=966 \mathrm{~mm}, \alpha=40^{\circ}, O D D=75 \mathrm{~mm}$ and $D_{R O I}=90 \mathrm{~mm}$. 


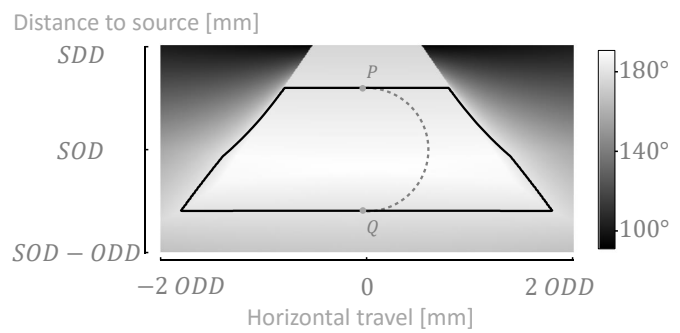

(a)

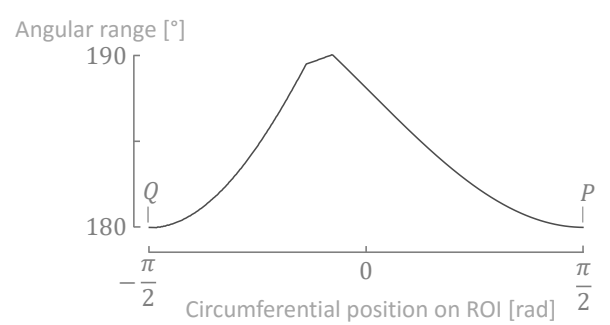

(b)

Figure 4: (a) The angular range covered by the points in a rectangular region containing the ROI. The thick black contour contains all of the points which cover at least $180^{\circ}$, including the ROI studied here; (b) By following the ROI circumference (dashed line in (a)) it can be seen that the points $P$ and $Q$ do indeed cover an angular rang of $180^{\circ}$, while the other points cover a larger range. (cfr. figure $3, L=966 \mathrm{~mm}, \alpha=40^{\circ}, O D D=75 \mathrm{~mm}$ and $D_{R O I}=90 \mathrm{~mm}$ ).

In principal, only the $O D D$ and $D_{R O I}$ are known a priori, indeed given the sample's diameter $D_{s}$, the following most hold

$$
\begin{aligned}
& D_{R O I} \geq D_{s} \\
& O D D \geq \frac{D_{s}}{2}
\end{aligned}
$$

In other words, the sample should be contained within the ROI and should not hit the detector plane. $L$ and $\alpha$ have to be chosen but in practice will be dictated by a certain demand in throughput (see also paragraph 2.2) and technical limitations such as the detector size and source's collimator opening. Moreover, the fixed nature of either one $(L$ or $\alpha)$ can be interchanged for a specific choice of $S D D$ or $S O D$, as long as equation 6 to 8 are fulfilled.

\subsection{Influence of the geometrical parameters on throughput}

At first glance there seems to be no apparent reason to choose a large horizontal travel $H$, specifically because this requires a larger and thus more expensive detector. Moreover, the average X-ray flux $\left(I_{a v}\right)$ seen by the sample will quickly drop off for higher $H$ according to (see the appendix)

$$
I_{a v}=I_{r e f} \cdot \frac{S D D_{r e f}^{2}}{S D D^{2}} \cdot f(x)
$$

given

$$
f(x)=\frac{\arctan (x)}{x} \quad \text { for } \quad x=\frac{H}{2 S O D}
$$


and where $I_{r e f}$ is the X-ray flux on the central ray measured at a reference distance $S D D_{\text {ref }}$ from the X-ray source. Following equations 6 to 8 , a larger $H$ will lead to an on average less favourable SNR performance, primarily because of the quadratic intensity decrease caused by an increasing $S D D$, while $f(x)$ remains practically constant. Hence, in order to maintain a constant SNR, the reference flux $\left(I_{r e f}\right)$ has to be increased, for example by increasing the tube power. A larger $H$ can however be advantageous considering more samples can be scanned simultaneously with a larger detector field. In the limiting case of a parallel beam $(S D D \rightarrow \infty)$ the projections of subsequent samples will not overlap and the number of samples which can be scanned simultaneously $\left(N_{s}\right)$ is given by

$$
N_{s}=\frac{L}{D_{R O I}}
$$

However, for a real case, the cone beam can produce overlapping projections at the edges of the sample's translation path when the distance between two subsequent samples is too small. The minimal distance between the samples can be derived from figure 2(b), which depicts the start of a new acquisition. Here, the tangent ray in point $P$ delineates the contours of the sample's projection on the detector, and dictates how close a preceding sample (not depicted in figure 2(b) can be to the new sample. In other words, the tangent through $P$ forms a mutual tangent between the ROI's of subsequent samples at the start of a new acquisition. The minimal distance between these samples is

$$
\Delta_{\min }=\frac{D_{R O I}}{\sin (\Theta)}
$$

and thus for the number of samples which can be imaged simultaneously the following holds

$$
N_{s}=\frac{H}{D_{R O I}} \cdot \sin (\Theta)
$$

The effective throughput $\left(T_{\text {eff }}\right)$, as in the number of samples which can be scanned per second, for the conveyor belt setup is then given by

$$
T_{\text {eff }}=\frac{N_{s}}{N_{p} \cdot t_{\text {exp }}} \quad\left[\frac{\text { samples }}{\text { sec }}\right]
$$

with $N_{p}$ the number of projections acquired during the scan at an exposure time of $t_{\text {exp }}$ seconds for a single projection. 


\section{Materials \& Methods}

\subsection{Simulation of conveyor belt scans}

140 with a specific choice for $L, \alpha, O D D$ and $D_{R O I}$. In most practical cases only the detector length $L$ or more specifically the travel $H$ will vary, since $O D D$ and $D_{R O I}$ are determined by the sample diameter $D_{s}$ and $\alpha$ will be limited by the source collimator opening or by a sensible limit posed upon the detector run-out $W$. Hence, in the following we consider $H$ to be the only variable, geometrical parameter, while the other parameters will be either fixed at a certain value or calculated through equations 6 to 8 . Given a fixed exposure time $\left(t_{\text {exp }}\right)$, the number of projections $\left(N_{p}\right)$ then completely defines a conveyor belt acquisition, which together with $H$ leaves two independent variables for the characterization of the image quality produced by a conveyor belt setup. Although the problem of characterizing the image quality for conveyor belt scans is now reduced to sweeping a two dimensional $\left(H, N_{p}\right)$ parameter space, it is still difficult to do this in a real life setup, as several other parameters indirectly vary with $H$ through equation 6 to 8 . However, as explained in the next paragraph, an effort has been made to build a flexible mock-up, which can mimic a conveyor belt acquisition for a broad range of $\left(H, N_{p}\right)$-pairs.

To further extend this range an X-ray radiograph simulator was used to produce realistic simulations of conveyor belt acquisitions [16]. This projection simulator implements the complete dependency of a radiograph on the X-ray energy spectrum by incorporating the X-ray source spectra, the energy dependent detector response and the energy dependent attenuation prescribed by a digital, three dimensional phantom. While the detector response and the source spectra can be selected out of a series of presets, which mimic the detectors and sources available at the "Centre for X-ray Tomography" of the Ghent University (UGCT; www.ugct.ugent.be), the digital phantom has to be constructed by composing a three dimensional grid of voxels each being assigned to a material with a specific energy dependent attenuation. For the simulations, a realistic phantom was extracted out of an X-ray micro-CT $(\mu \mathrm{CT})$ scan (voxel size $64 \mu \mathrm{m}$ ) of an Elstar apple. The same sample was also used to study the performance of the experimental

mock-up. The gray values in this high resolution scan were used to assign each voxel to a certain density class of apple tissue which was modelled as a soft tissue material [17] with a mass density scaled according to the gray value of each class. The scaling was performed in such a way that air is rep- 
resented by the lowest level, corresponding to a density of $1.2 \cdot 10^{-3} \mathrm{~g} / \mathrm{cm}^{3}$ [18, while the average attenuation value $\mu_{b u l k}=0.29 \mathrm{~cm}^{-1}$ calculated for the bulk apple tissue segmented from the high resolution scan, is associated to the measured mass density of the apple tissue, i.e. $\rho_{\text {apple }}=0.84 \mathrm{~g} / \mathrm{cm}^{3}$. Indeed, by segmenting the apple tissue while excluding the core air space, it is possible to accurately determine the tissue volume and subsequently its mass density by weighing the apple. The procedure for constructing a digital phantom is summarized in figure 5 .

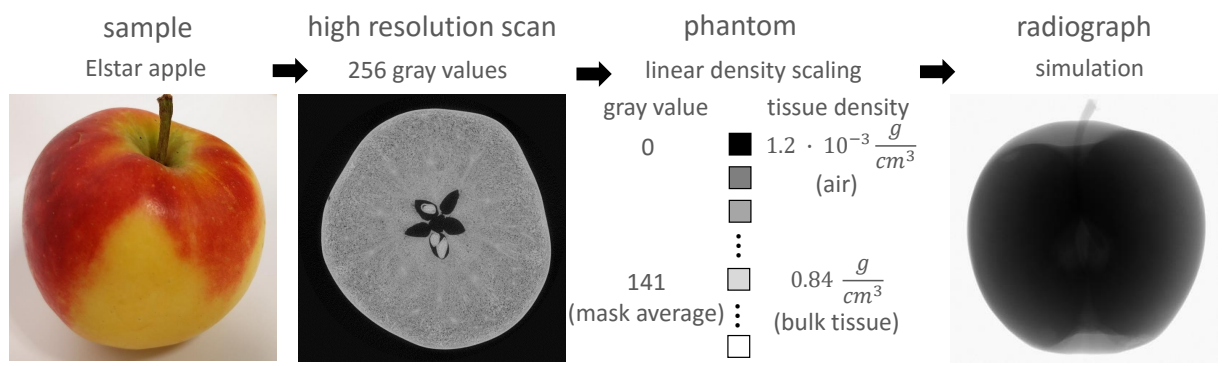

Figure 5: Procedure for constructing a digital phantom, as an input to the radiograph simulator. The gray values from an X-Ray $\mu \mathrm{CT}$ scan (voxel size $64 \mu \mathrm{m}$ ) of an Elstar apple are used to represent 256 apple tissue classes, which are modelled by a soft tissue mass attenuation curve and a density linearly scaled to their gray values.

\subsection{A prototype conveyor belt setup}

An existing lab based X-ray micro-CT setup at the UGCT (figure 6) was equipped with an add-on module containing a flat panel detector (Varian Medical Systems GmbH, Willich, Germany) and an extra rotation stage (PI miCos GmbH, Freiburger, Germany). The detector and the rotation stage are fixed w.r.t. each other such that the distance between the detector surface and the rotation axis remains constant, i.e. $O D D=84.5 \mathrm{~mm}$. The add-on module is mounted on a translation stage which is normally used to select one of the detectors from the setup and to align it with the X-ray source and the original rotation axis of the setup. The combined movement of the translation stage and the rotation stage makes it possible to mimic conveyor belt trajectories with a travel limited to the translation stage's spindle length $\left(H_{\max }=556 \mathrm{~mm}\right)$. The translation stage in turn is mounted on another motorized stage which can set the $S D D$. The three axes of motion allow for practically any conveyor belt acquisition. However, some geometrical parameters are implicitly fixed, such as the detector run-out $(W)$ which is 
given here by the detector width, the ROI diameter set to the maximal diameter of the sample $\left(D_{s}\right)$ and subsequently the half opening angle $(\alpha)$, by taking into account equation 1 . The opening angle is well within the limits of the setup, as here an uncollimated transmission type X-ray source (X-RAY WorX GmbH, Garbsen, Germany) was used, which radiates quasi-uniformly from its source point. An overview of the geometrical parameters and their fixed nature is given in table 1. In order to minimize the drying and the build-up of browning disorders in the apple sample, all of the scans were performed on the same day, including the high resolution scan, which was used to construct the digital phantom.

Table 1: Overview of the geometrical parameters of the conveyor belt mock-up. Only the horizontal travel $(H)$ is varied independently, while the source detector distance $(S D D)$ and the rotation angle $(\Theta)$ are calculated from equations 6 to 8 . The other parameters are fixed by design.

\begin{tabular}{llccccc}
\hline \hline & \multicolumn{5}{c}{ Fixed? } & Value $\backslash$ Range \\
\hline horizontal travel & $(H)$ & no & -278 & $\ldots$ & 278 & $\mathrm{~mm}$ \\
source detector distance & $(S D D)$ & no & 200 & $\ldots$ & 1300 & $\mathrm{~mm}$ \\
rotation angle & $(\Theta)$ & no & no restrictions & $\circ$ \\
ROI diameter & $\left(D_{R O I}\right)$ & yes & 65 & $\mathrm{~mm}$ \\
object detector distance & $(O D D)$ & yes & 84.5 & $\mathrm{~mm}$ \\
detector run-out & $(W)$ & yes & 145.5 & $\mathrm{~mm}$ \\
half opening angle & $(\alpha)$ & yes & 24 & $\circ$ \\
detector pixel size & $\left(p_{d}\right)$ & yes & 254 & $\mu \mathrm{m}$ \\
\hline \hline
\end{tabular}

\subsection{Quality assessment of CT reconstructions}

Iterative $C T$ reconstruction. For standard circular cone beam and helical cone beam acquisitions the reconstruction can be performed through fast analytical reconstruction algorithms, i.e. the Feldkamp-David-Kress (FDK) [19] and Katsevich [20] algorithms, respectively. An essential prerequisite for the FDK algorithm is that the projections be sampled in an equiangular fashion on a circular trajectory, which is not the case for the conveyor belt setup presented in this work. By introducing an angular remapping of the projections and by applying an appropriate weighting during the reconstruction's back projection step, the FDK algorithm can be used to perform a reconstruction of a conveyor belt acquisition. In order to handle the uncommon geometry of the conveyor belt setup and to avoid the remapping 


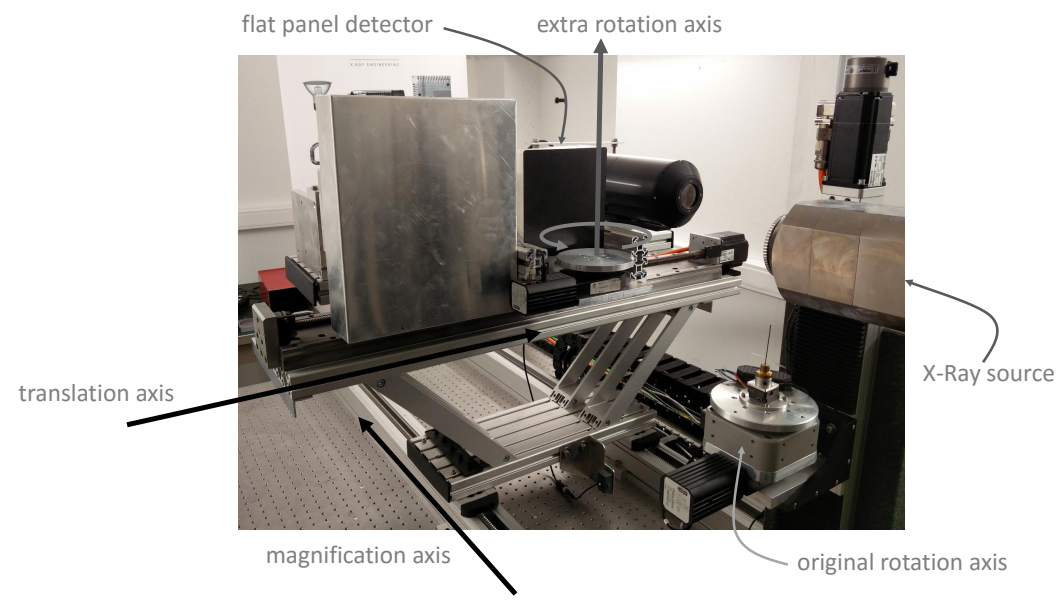

Figure 6: An existing X-ray micro-CT setup was equipped with an extra detector and a co-moving rotary stage. The module containing this detector and rotary stage is mounted on a translation axis, which is normally used to align one of several detectors on this setup with the X-ray source. The translation stage in turn is mounted on another axis which sets the $S D D$.

and reweighting steps, an iterative reconstruction scheme is used, specifically the simultaneous algebraic reconstruction technique (SART) [21], which has proven to yield an optimal balance between reconstruction speed and stability [22]. Iterative schemes are typically more computationally demanding, but are more extendible and flexible towards the implementation of alternative geometries, a priori information about the imaged sample and advanced models for imaging process itself [23, 24]. An in-house software tool was developed to handle projections taken from virtually any acquisition geometry and to reconstruct them into a 3D volume, by implementing a version of SART in python. The computationally demanding tasks, however, are offloaded to a graphical processing unit (GPU) through the PyCUDA python module [25], offering a tremendous decrease in reconstruction times.

Spectral Signal to Noise Ratio (SSNR). The quality of the reconstructions was quantified by calculating their apparent resolution, through a Spectral Signal-to-Noise Ratio (SSNR) [26, 27]. The SSNR is calculated by centring the 3D Fourier transforms of two volumes at their zero frequency, i.e. a reconstruction of the projections taken from a sample on the one hand and a reconstruction based on projections containing only noise on the other. Subsequently, the power of the 3D Fourier signals is averaged out over a 
series of spherical shells built up from the centre, which essentially reduces a $3 \mathrm{D}$ signal to $1 \mathrm{D}$ signal representing the power spectra, denoted by $F_{d}\left(f_{R}\right)$ for the data reconstruction and by $F_{n}\left(f_{R}\right)$ for the noise-only reconstruction. The SSNR is then given by

$$
\operatorname{SSNR}\left(f_{R}\right)=\max \left(\frac{F_{d}\left(f_{R}\right)}{F_{n}\left(f_{R}\right)}-1,0\right)
$$

where $f_{R}$ is the frequency radius of the spherical shells. A cut-off can be set on the resulting SSNR-curve, representing the minimal signal-to-noise ratio $\left(S N R_{\text {min }}\right)$ necessary for a frequency component to be distinguishable from its noisy background. Here,

$$
{ }^{2} \log \left(1+S N R_{\min }\right)=0.5
$$

assuring that the average information content of a voxel in Fourier space is at least $1 / 2$ bit [28, 29]. It should be noted that following the definition of the SSNR, the SNR in this work is defined as the ratio of the variance to the square mean value of a signal, i.e. $S N R=\sigma^{2} / \mu^{2}$. The frequency at which the SSNR-curve reaches the cut-off $S N R_{\min }$ can be interpreted as the maximal frequency $\left(f_{\max }\right)$ which is adequately represented by the reconstructed volume, and hence its inverse $\left(f_{\text {max }}^{-1}\right)$ serves as a resolution measure indicating the minimal feature sizes visible on the reconstruction.

\section{Results}

\subsection{Simulated scans versus mock-up scans}

A series of conveyor belt scans was acquired through both simulation and real life acquisitions with the mock-up setup discussed in paragraph 3.2 . Moreover, the scans which were performed with the mock-up conveyor belt were also simulated, making it possible to compare both. But while the travels $(H)$ for the mock-up are limited by design, larger travels can still be simulated. Each of the setups listed in table2, were executed five times for a varying number of projections, going from 100 to 500 in steps of 100, leading to 20 scans acquired with the mock-up and 35 simulated scans in total.

For table 2 it should be noted that the detector illumination $\left(t_{\text {exp }} \cdot P\right)$ is varied in order to completely fill up the detector's dynamic range as to compensate for the quadratic drop in the X-ray flux with the increasing $S D D$. In the mock-up setup this was achieved by altering the exposure 
Table 2: Overview of the scans performed with the mock-up and the simulated scans. The travel $(H)$ is varied while the $S D D, L$ and $\Theta$ are calculated through equations 6 to 8 . The detector illumination is expressed as a product of the exposure time and the tube power. The tube high voltage was set to $120 \mathrm{kV}$.

\begin{tabular}{|c|c|c|c|c|c|c|}
\hline $\begin{array}{c}\text { Simulated } \\
\text { scans }\end{array}$ & $\begin{array}{c}\text { Mock-up } \\
\text { scans }\end{array}$ & $\begin{array}{c}H \\
{[\mathrm{~mm}]}\end{array}$ & $\begin{array}{c}L \\
{[\mathrm{~mm}]}\end{array}$ & $\begin{array}{l}S D D \\
{[\mathrm{~mm}]}\end{array}$ & $\begin{array}{c}\Theta \\
{[0]}\end{array}$ & $\begin{array}{c}t_{\text {exp }} \cdot P \\
{[W s]}\end{array}$ \\
\hline $\mathrm{V}$ & $\mathrm{V}$ & 200 & 318.7 & 361.5 & 153.0 & 0.800 \\
\hline $\mathrm{V}$ & $\mathrm{V}$ & 300 & 416.4 & 472.4 & 146.7 & 1.368 \\
\hline V & $\mathrm{V}$ & 400 & 515.2 & 584.4 & 143.3 & 2.092 \\
\hline $\mathrm{V}$ & $\mathrm{V}$ & 500 & 614.4 & 697.0 & 141.2 & 2.976 \\
\hline$\overline{\mathrm{V}}$ & & $60 \overline{0}$ & $\overline{7} 13.9$ & $\overline{80} \overline{9} . \overline{9}$ & $\overline{13} \overline{9} . \overline{9}$ & 4.016 \\
\hline $\mathrm{V}$ & & 700 & 813.5 & 922.9 & 138.8 & 5.216 \\
\hline $\mathrm{V}$ & & 800 & 913.2 & 1036.0 & 138.0 & 6.568 \\
\hline
\end{tabular}

time $\left(t_{\text {exp }}\right)$, because the transmission X-ray tube used in the setup had a limited output power, here set to $4 \mathrm{~W}$. The power limitation is not an issue for industrial, directional tubes, which can reach up in the order of $1 \mathrm{~kW}$ in tube power. So in a practical setup, rather than the detector's exposure time, the power would be adjusted towards filling up the dynamic range of the detector.

In figure 7, three projections of the sample, moving along its conveyor belt trajectory, are superimposed on what would be the full field of view of a stationary detector of length $L=319 \mathrm{~mm}$, corresponding to a travel of $H=200 \mathrm{~mm}$. Based on a visual comparison a good agreement was found between the mock-up scans and the simulated scans, while some small differences can clearly be seen on the line profiles for transmission and the local SNR, following a horizontal line through the centre of the middle projection (indicated in figure 7). These small difference are to a large part explained by a slight misalignment between the digital phantom and the real sample. Most importantly, the SNR is very similar in both cases, which is crucial with respect to the quality of the $\mathrm{CT}$ reconstructions.

The reconstructions are performed on a grid of 545 slices containing 570 by 570 cubical voxels with a volume of $128^{3} \mu \mathrm{m}^{3}$ each, of which the central slice is shown in figure 8 for $H=200 \mathrm{~mm}$. Visually the simulated and mockup reconstructions proved to be very similar, although the simulated scans appear to be slightly sharper. This can also be seen in the histograms, where some of the features are smoothed out in the mock-up reconstructions. Most 
importantly, the overall sharpness improves as the number of projections $\left(N_{p}\right)$ is increased. This effect can be leveraged when setting a larger travel $(H)$, which allows for more projections to be acquired at a similar throughput, and for smaller features to be visualised. This gain in sharpness is barely visible to the naked eye, and is therefore not depicted in figure 8, though it can be quantified through the SSNR (see figure 9p. However, at a fixed number of projections, there is loss in sharpness as the travel is increased, primarily caused by a decrease in the geometrical magnification for larger $S D D$ 's at a constant $O D D$, next to a slight drop in the factor $f(x)$ in equation 10 . which in these acquisitions was not compensated for by setting a higher illumination.

\subsection{Quality as a function of throughput}

290 In paragraph 3.3 , the SSNR was introduced as means to measure the smallest features which can be detected in the reconstructions of the conveyor belt acquisitions. It is particularly interesting to look at this minimal feature size in terms of the throughput realised by the conveyor belt system, calculated according to equation 11 for a given exposure time, in this case $20 \mathrm{~ms}$ for a single projection, which is a minimum for the detector used in the mock-up (Varian Medical Systems GmbH, Willich, Germany). Looking at figure 9 , it is clear that the image quality produced by the mock-up is not as good as for the simulated scans, in the sense that the simulated scans might be overestimating quality. Nevertheless, the general trends as a function of the horizontal travel and the number of projections are reproduced in both the simulated and the mock-up case, apart from one anomaly occurring at a horizontal travel of $200 \mathrm{~mm}$ for the mock-up. Specifically, as the number of projections increases the detectable feature size significantly decreases, with the drawback of longer acquisition times and thus a lower throughput. This drop in throughput can be countered by increasing the horizontal travel, causing the curves to slowly shift towards higher throughputs. To summarize figure 9, small features can only be detected in a fast way through larger setups. Finally, it is worthy to note that the minimal feature size calculated through the SSNR is up to seven times larger than the voxel size (here 128 $\mu \mathrm{m})$ of the reconstruction grids, in sense that a voxel size is not always a fair reflection of the underlying resolution. The double of the voxel size (256 $\mu \mathrm{m})$ does however constitute an absolute minimum for the resolution, such that for a very large number of projections the curves in figure 9 should theoretically converge towards this value. It should also be noted that the 

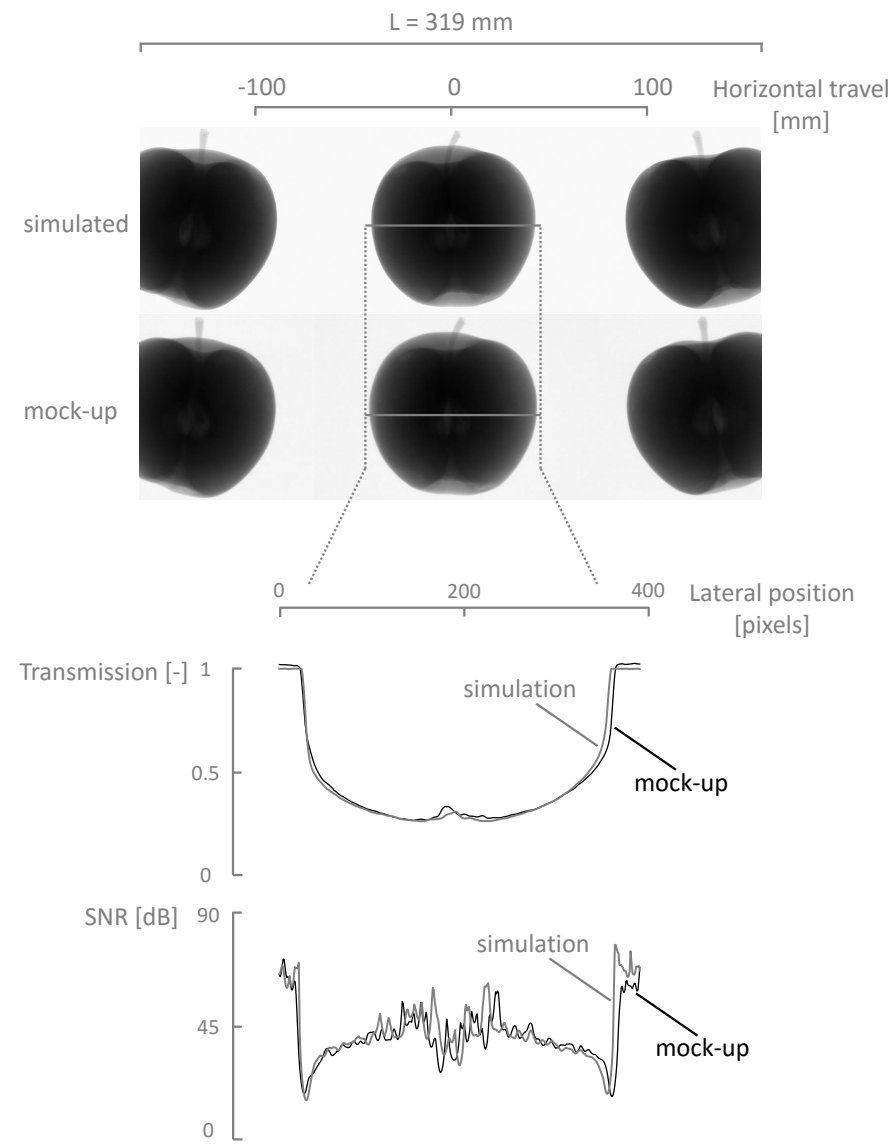

Figure 7: Comparison between three projections acquired with the mock-up conveyor belt and their simulated counterparts (top). The projections are shown from left to right as they would be acquired on a stationary detector with a span $L$, here $L=319 \mathrm{~mm}$ for $H=200 \mathrm{~mm}$. Although the overall agreement is good, some small differences, which can be attributed to slight misalignments between the digital phantom and the real apple, are visible on line profiles for the transmission and the local SNR (bottom).

voxel size of $128 \mu \mathrm{m}$ was chosen to be smaller than the detector's pixel pitch $p_{d}=254 \mu \mathrm{m}$, such that the lower limit on the resolution estimates calculated according to equation 12 is primarily governed by $N_{p}$ rather than the voxel size itself. According to the lower bound on figure 9(b) the voxel size for the reconstruction grids can chosen to be larger in practice, i.e. approximately ${ }_{320} p_{v} \approx 250 \mu m$ (see also equation 13 . 


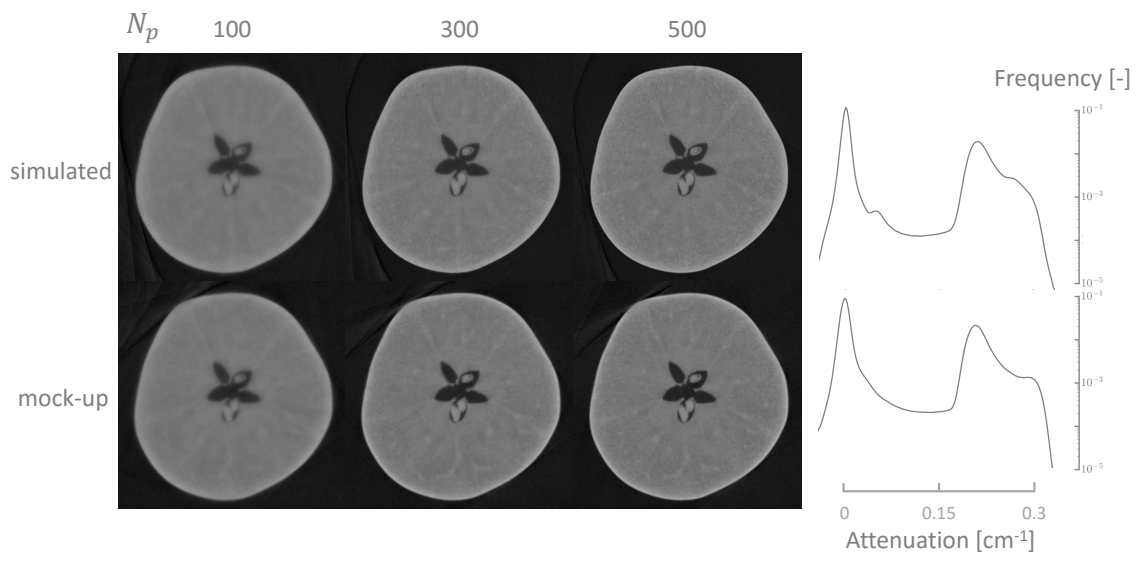

Figure 8: Reconstruction for the central slice in the Elstar apple based on the simulated (top) and the mock-up projections (bottom) for a varying number of projections $\left(N_{p}\right)$ and $H=200 \mathrm{~mm}$. The simulated scans seem to be sharper, and as expected the sharpness increases significantly with $N_{p}$. The histograms for the $N_{p}=500$ case (right) are very similar, apart some feature which seem to be smoothed out in the mock-up reconstructions.

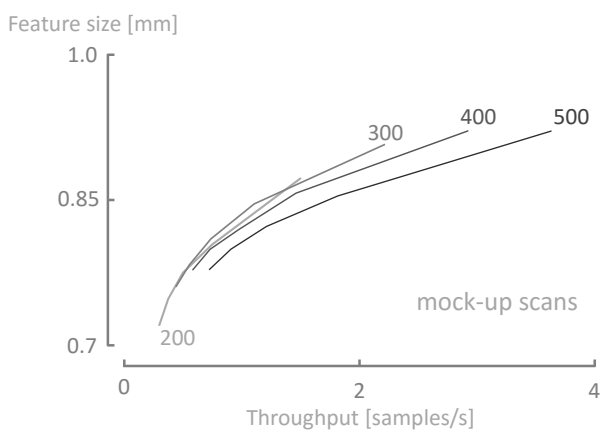

(a)

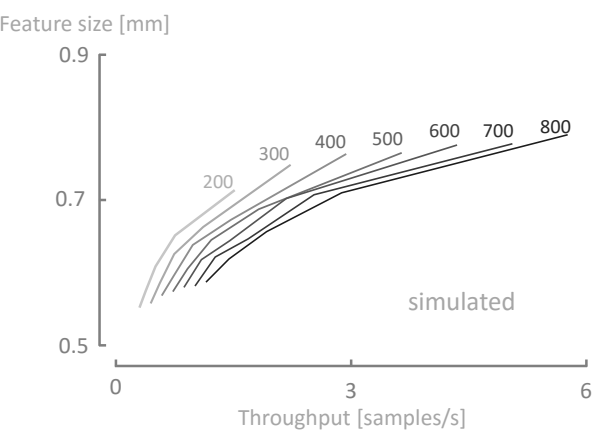

(b)

Figure 9: The detectable feature sizes as a function of the throughput for the mock-up scans (a) and the simulated scans (b). Each curve represents a different travel as indicated next to the curve in 'mm'. The number of projections increases from 100 to 500 following each curve from right to left, showing a clear trend towards smaller feature sizes for slower scanning regimes. 


\section{Discussion}

\subsection{Towards simulation based design}

An analysis, such as the one outlined in this work, can be performed purely on a simulation basis, but care needs to be taken in drawing conclusions 325 as the mismatch between the simulations and the mock-up case in figure 9 points out. Several sources of uncertainties are not accounted for in the simulations, and can to a large part explain the loss in resolution encountered in the real data. In order of importance:

- Sample movement and small calibration errors in the real life sample trajectories can cause excessive blurring in the resulting reconstructions. Hence, when a conveyor belt system is commissioned, a careful mechanical calibration of the sample trajectories and/or an algorithmic strategy to compensate for positioning errors during reconstruction, will be necessary.

- Systematic downward offsets with respect to the tube power setpoint can cause an illumination shortage, and subsequently a lower SNR. Together with the aforementioned point, the authors believe this might partly explain the shift of the $H=200 \mathrm{~mm}$ curve with respect to the other curves on figure 9 in the mock-up case. This is why the tube power will be carefully logged in follow up experiments.

- Photon scattering caused by the sample itself, which is particularly a problem for small ODD's where the scattering footprint forms a smeared out halo like structure around the projection of the sample.

- Cross talk between the detector pixels caused by an oblique entrance of X-rays might impose a practical limit upon the cone angle and the detector length, next to a degradation of resolution.

As mentioned before, a slight improvement in image quality can be achieved while maintaining a similar throughput by increasing the horizontal travel, or vice versa a higher throughput can be achieved for the same image quality. There are however other ways, next to larger travels, to increase throughput, e.g. by introducing multiple inspection stations in parallel, or even better, by considering the fact that several samples might simultaneously fit into the reconstruction region depicted on figure 4(a). As such, the shape of this 
reconstruction region and the way it is filled up by the sample, is equally important in the design phase. For instance, the ROI diameter might deliberately be chosen larger than the sample such that the reconstruction region can indeed contain several samples at once. The shape of the reconstruction region can also be tailored by introducing a whole series of alternative conveyor belt like trajectories through a generalization of the mapping between the rotational and the translational movement.

\subsection{Generalized conveyor belt like trajectories}

When considering a counter clockwise rotation, the rotation angle $(\theta)$ can follow any continuous mapping $(g)$ along the horizontal travel coordinate $(h)$, which can be expressed as follows

$$
g: h \rightarrow \theta \quad h \in\left[-\frac{H_{\text {out }}}{2}, \frac{H_{\text {out }}}{2}\right]
$$

Subject to

$$
\left|g\left( \pm \frac{H^{\prime}}{2}\right)\right| \geq \Theta^{\prime} \text { and }\left|g\left( \pm \frac{H_{\text {out }}}{2}\right)\right| \geq \Theta^{\prime}
$$

with

$$
H_{\text {out }}=H^{\prime}+\frac{2 D_{R O I}}{\cos (\alpha)}
$$

representing the distance between the extremal position of the sample, where its ROI is just outside of the detector's field of view, tangent to the extremal source rays. In words, the conditions on

state that there should be at least one ray tangent to the ROI for $P$ and $Q$ on both the left and the right side of the central ray. This opens up a wide range of possible trajectories and embodiments for a conveyor belt geometry. The one focused upon in this work follows equation 9, where both the acquisition and the rotation are initiated at position $h=-H / 2$ as in figure 2(b). In a similar implementation the acquisition could be started together with the translation at $h=-H_{\text {out }} / 2$, while the rotation would only start at $h=-H^{\prime} / 2$, eventually performing an identical rotation of $2 \Theta^{\prime}$ between $-H^{\prime} / 2$ and $H^{\prime} / 2$. These trajectories can be realized in practice by attaching a sample tray to a pinion which in turn grabs into a linear rack 
gear, producing a simultaneous rotation around the pinions axis and a linear translation parallel to the rack. It is clear that all of the conveyor belt like trajectories introduce an additional translational movement with respect to

the traditional circular cone beam acquisitions. To that end it is important to note that the projections of some points in the sample, more than others, may be subject to motion blurring when they are shifted by more than one detector pixel during one exposure time. Hence, an important question to be investigated in future work, is whether the generalized conveyor belt like trajectories offer a better reconstruction quality and most importantly whether they might provide larger reconstruction regions (cfr. figure 4(a)) while causing less motion blurring overall.

\subsection{Detector \& X-ray tube properties}

In order to perform a qualitative CT scan a good detector and X-ray tube are essential. Although their properties have to be tuned to the application at hand, there are some general guidelines which can be followed in the case of a conveyor belt setup, making it possible to extrapolate the methods discussed here to other types of samples. First of all, it should be noted that the conveyor belt setup is meant to be operated in a so called 'focal spot demagnification' regime, meaning that the $O D D$ is typically smaller than the $S O D$. The advantages of demagnification are that:

1. the span of the cone beam is larger closer to the detector, and as such more product samples can be imaged simultaneously.

2. the X-ray spot size will be demagnified by a factor $M_{s}=O D D / S O D$, which allows for larger spot sizes $\left(p_{s}\right)$ than the detector pixel size $\left(p_{d}\right)$. As a consequence, a higher tube power can be set, and the heat generated in the tube's focal spot can be dissipated across a larger area.

Unfortunately, a demagnification of the focal spot also implies that there will barely be any object magnification $\left(M_{o}\right)$ and that the voxel size of the 3D CT reconstruction $\left(p_{v}\right)$ will be more or less equal and limited to the detector's pixel pitch, according to

$$
p_{v}=\frac{1}{M_{o}} p_{d}+\frac{M_{s}}{M_{o}} p_{s}
$$

where $p_{s} \leq p_{d} / M_{s}$, i.e. the demagnified spot size should be smaller than the detector pixel size. Hence, choosing a detector pixel size indirectly imposes 
an upper limit on the X-ray focal spot size, while the latter limits the power which can be deposited in the focal spot area. Typically, the target of an $\mathrm{X}$-ray tube can safely dissipate a maximum of $1 \mathrm{~kW}$ per $\mathrm{mm}$ of focal spot diameter, which leads to the following coincidental relation between the tube power $(P)$ and the detector pixel size

$$
P[W] \leq \frac{p_{d}[\mu m]}{M_{s}}
$$

For the cases discussed here a detector with a pixel pitch of $0.254 \mathrm{~mm}$ was simulated in a setting were the X-ray focal spot is demagnified by a factor of at most $M_{s}=0.23$, which implies a maximal focal spot size of approximately $1.1 \mathrm{~mm}$, and subsequently a maximum tube power of about $1.1 \mathrm{~kW}$. This tube power is quite large, in the sense that the dynamic range of most detectors will be clipped well before reaching this limit, even at very low exposure times. As it is implicitly assumed that the detector is read out as fast as possible in order to reach a high throughput (cfr. equation 11), the tube power is actually determined by the detector's saturation limit rather than any geometrical restrictions on the focal spot size.

The SNR in the resulting CT reconstruction can also be optimised by setting an optimal tube high voltage. Again this parameter is highly dependent on the type and size of the sample, but can be estimated by looking at the theoretical attenuation through a slab with a material and thickness representative for the sample under study, e.g. the Elstar apple can be modelled as a $6 \mathrm{~cm}$ thick slab of soft-tissue with a density of $0.8 \mathrm{~g} / \mathrm{cm}^{3}$. The theoretical transmission through this slab can be calculated across several energy bins covering the complete X-ray tube's spectrum, followed by a back projection of the attenuating mass and its associated error into a resolution element representative of a CT reconstruction [16, 30]. The end result is a curve of the expected SNR as a function of the X-ray tube's high voltage at a fixed output power, which clearly shows a maximum at $120 \mathrm{kV}$ for the sample studied here (figure 10.

\section{Conclusion}

It was shown that under certain mathematical constraints the combination of a translational and rotational sample movement can produce a series of radiographs from which a theoretically exact CT reconstruction can be ob-

tained, leaving small cone beam artefacts for off-centre slices aside. Within 


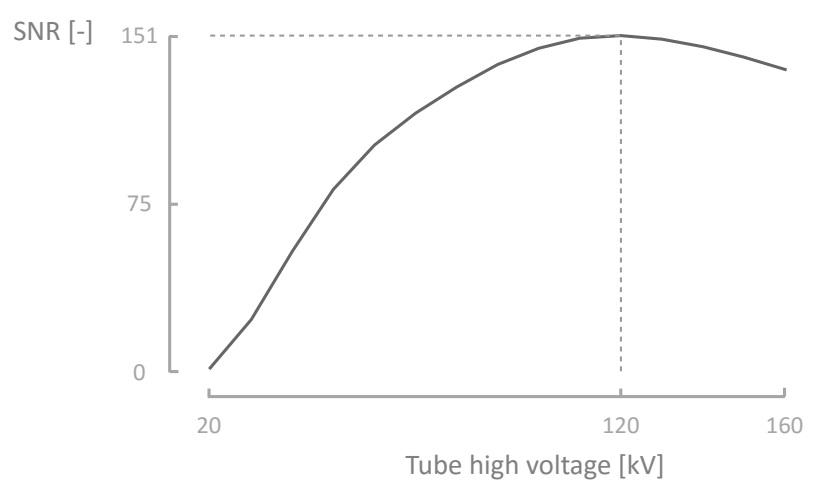

Figure 10: The estimated SNR on the calculated attenuation value of a $6 \mathrm{~cm}$ slab of soft tissue as a function of the X-ray tube's high voltage. A maximum for the SNR can be found at $120 \mathrm{kV}$, which subsequently serves as an optimal tube voltage for this sample at a given tube power.

the boundaries of these mathematical constraints a methodology to evaluate the design of a conveyor belt system was built up, which may serve as a tool to tailor and optimize the design of the system toward a specific type of sample, i.e. other than the Elstar apple. As an end result to the design exercise plots can be generated, which visualize the trade-off between the minimal feature sizes which need to be detected, and how fast they have to be imaged, i.e. the throughput as a function of the size of the system and the duration of an acquisition, in terms of the horizontal travel and the number of projections, respectively. In summary to the plots, generated here for the Elstar apple sample in both a simulation and mock-up environment (figure 9), it can be concluded the throughput for this particular case tends to a realistic, practically usable range of up to 5 samples per second. In general, for different types of samples, the analysis performed here indicates that larger setups can achieve a higher throughput, at the same level of image quality. Furthermore, other ways to improve throughput remain to be investigated in future work, primarily the tailoring of the reconstruction region's shape by looking at alternative conveyor belt like sample trajectories. In conclusion, the concept of a conveyor belt acquisition geometry is shown to be a viable solution to in-line non-destructive testing with 3D X-ray CT in a continuous high throughput environment. 


\section{Appendix}

Looking at figure 1, the distance between the source point and any given point on the central detector row can be written as a function of the travel position $(h)$

$$
R(h)=\sqrt{S D D^{2}+h^{2}}
$$

As the X-ray intensity decreases quadratically with an increasing distance from the source point

$$
I(h)=I_{r e f} \frac{S D D_{r e f}^{2}}{R(h)^{2}}
$$

the average intensity can be calculated by solving the following integral

$$
I_{a v}=I_{\text {ref }} \cdot S D D_{\text {ref }}^{2} \cdot \int_{-H M / 2}^{H M / 2} d h / R(h)^{2}
$$

where $M=S D D / S O D$ is the magnification, leading to equation 10 .

\section{Acknowledgments}

This work is supported by the Flemish agency for Innovation by Science and Technology under the TomFood project grant IWT SBO 120033 (www. tomfood.be). The Special Research Funds (BOF) of the Ghent University is acknowledged for the post-doctoral grant to M.N. Boone.

\section{References}

[1] Haff RP, Toyofuku N. X-ray detection of defects and contaminants in the food industry. Sensing and Instrumentation for Food Quality and Safety 2008;2(4):262-73. doi:10.1007/s11694-008-9059-8.

[2] Hanke R, Fuchs T, Uhlmann N. X-ray based methods for non-destructive testing and material characterization. Nuclear Instruments and Methods in Physics Research Section A: Accelerators, Spectrometers, Detectors and Associated Equipment 2008;591(1):14-8. doi:10.1016/j.nima. 2008.03 .016 .

[3] Kotwaliwale N, Singh K, Kalne A, Jha SN, Seth N, Kar A. X-ray imaging methods for internal quality evaluation of agricultural produce. Journal of food science and technology 2014;51(1):1-15. doi:10.1007/ s13197-011-0485-y. 
[4] Zou Y, Du D, Chang B, Ji L, Pan J. Automatic weld defect detection method based on Kalman filtering for real-time radiographic inspection

口 of spiral pipe. NDT \& E International 2015;72:1-9. doi:10.1016/j. ndteint.2015.01.002.

[5] Xu J, Liu T, Yin X. Automatic X-ray crack inspection for aircraft wing fastener holes. 2nd International Symposium on NDT in Aerospace $2010 ;: 1-8$.

[6] Herremans E, Melado-Herreros A, Defraeye T, Verlinden B, Hertog M, Verboven $\mathrm{P}$, et al. Comparison of X-ray CT and MRI of watercore disorder of different apple cultivars. Postharvest Biology and Technology 2014;87:42-50. doi:10.1016/j.postharvbio.2013.08.008.

[7] Kondo N. Robotization in fruit grading system. Sensing and Instrumentation for Food Quality and Safety 2009;3(1):81-7. doi:10.1007/ s11694-008-9065-x.

${ }_{480}$ [8] De Chiffre L, Carmignato S, Kruth JP, Schmitt R, Weckenmann A. Industrial applications of computed tomography. CIRP Annals - Manufacturing Technology 2014;63(2):655-77. doi:10.1016/j.cirp. 2014. 05.011 .

[9] Brunke O, Hansen F, Stuke I, Butz F. A new Concept for High-Speed atline and inlineCT for up to $100 \%$ Mass Production Process Control. Proceedings 18th World Conference on Non-Destructive Testing $2012 ;: 16-20$.

[10] Thompson W, Lionheart W, Morton E, Cunningham M, Luggar R. High speed imaging of dynamic processes with a switched source x-ray CT system. Measurement Science and Technology 2015;26(5):1-11. doi:10. 1088/0957-0233/26/5/055401.

[11] Warnett JM, Titarenko V, Kiraci E, Attridge A, Lionheart WRB, Withers PJ, et al. Towards in-process x-ray CT for dimensional metrology. Measurement Science and Technology 2015;27(3):035401. doi:10.1088/0957-0233/27/3/035401.

[12] Matenine D, Goussard Y, Després P. GPU-accelerated regularized iterative reconstruction for few-view cone beam CT. Medical physics 2015;42(4):1505-17. doi:10.1118/1.4914143. 
[13] Sidky EY, Kao CM, Pan X. Accurate image reconstruction from fewviews and limited-angle data in divergent-beam ct. Journal of X-ray Science and Technology 2006;14(2):119-39.

[14] Flores L, Vidal V, Verdú G. Iterative Reconstruction from Few-view Projections. Procedia Computer Science 2015;51:703-12. doi:10.1016/ j.procs.2015.05.188.

[15] Smith BD. Image reconstruction from cone-beam projections: necessary and sufficient conditions and reconstruction methods. IEEE transactions on medical imaging 1985;4(1):14-25. doi:10.1109/TMI .1985.4307689.

[16] Dhaene J, Pauwels E, De Schryver T, De Muynck A, Dierick M, Van Hoorebeke L. A realistic projection simulator for laboratory based Xray micro-CT. Nuclear Instruments and Methods in Physics Research Section B: Beam Interactions with Materials and Atoms 2015;342:170-8. doi:10.1016/j.nimb.2014.09.033.

[17] Berger MJ, Seltzer SM. XCOM Photon Cross Sections; vol. 3. National Institute of Standards and Technology Gaithersburg; 1999.

[18] Lemmon EW, McLinden MO, Friend DG. Thermophysical properties of fluid systems. NIST chemistry webbook, NIST standard reference database 2005;69.

[19] Feldkamp La, Davis LC, Kress JW. Practical cone-beam algorithm. Journal of the Optical Society of America A 1984;1(6):612. doi:10. 520 1364/JOSAA.1.000612.

[20] Katsevich A. Improved exact FBP algorithm for spiral CT. Advances in Applied Mathematics 2004;:32-681.

[21] Andersen AH, Kak AC. Simultaneous Algebraic Reconstruction Technique (SART): A Superior Implementation of the Art Algorithm. Ultrasonic Imaging 1984;6(1):81-94. doi:10.1177/016173468400600107.

[22] De Witte Y. Improved and practically feasible reconstruction methods for high resolution X-ray tomography. 2010. 
[23] Willemink MJ, de Jong PA, Leiner T, de Heer LM, Nievelstein RAJ, Budde RPJ, et al. Iterative reconstruction techniques for computed tomography Part 1: technical principles. European radiology 2013;23(6):1623-31. doi:10.1007/s00330-012-2765-y.

[24] Beister M, Kolditz D, Kalender WA. Iterative reconstruction methods in X-ray CT. Physica medica : PM : an international journal devoted to the applications of physics to medicine and biology : official journal of the Italian Association of Biomedical Physics (AIFB) 2012;28(2):94-108. doi:10.1016/j.ejmp.2012.01.003.

[25] Klöckner A, Pinto N, Lee Y, Catanzaro B, Ivanov P, Fasih A. PyCUDA and PyOpenCL: A scripting-based approach to GPU run-time code gen-

q eration. Parallel Computing 2012;38(3):157-74. doi:10.1016/j. parco. 2011.09.001.

[26] Penczek PA. Three-dimensional spectral signal-to-noise ratio for a class of reconstruction algorithms. Journal of Structural Biology 2002;138(12):34-46. doi:10.1016/\$1047-8477(02)00033-3.

[27] Unser M, Sorzano COS, Thévenaz P, Jonić S, El-Bez C, De Carlo 545 $\mathrm{S}$, et al. Spectral signal-to-noise ratio and resolution assessment of 3D reconstructions. Journal of structural biology 2005;149(3):243-55. doi: $10.1016 / j \cdot j s b .2004 .10 .011$.

[28] van Heel M, Schatz M. Fourier shell correlation threshold criteria. 1 Journal of structural biology 2005;151(3):250-62. doi:10.1016/j.jsb. 550 2005.05.009.

[29] Shannon CE. A mathematical theory of communication. ACM SIGMOBILE Mobile Computing and Communications Review 2001;5(1):3. doi:10.1145/584091.584093.

[30] Chesler DA, Riederer SJ, Pelc NJ. Noise due to photon counting statistics in computed X-ray tomography. Journal of computer assisted tomography 1977;1(1):64-74. 